\title{
LES STADES PRÉIMAGINAUX DES BLËPHAROCERIDES (DIPTERA, NEMATOCERA) DE CORSE. ETUDE TAXONOMIQUE ET ẼCOLOGIQUE
}

\author{
par Jean GiUdicelli.
}

\section{1. - INTRODUCTION}

La faune des Blépharocérides de Corse compte sept espèces. Six sont rangées dans la sous-famille des Blépharocerinae ce sont :

Dioptopsis vernus Giudicelli, 1963.

Liponeura bischoffi Edwards, 1928.

Liponeura cinerascens Loew, 1844.

Liponeura cortensis Giudicelli, 1963.

Liponeura edwardsiana Mannheims, 1954.

Liponeura insularis Giudicelli, 1965.

Apistomyia elegans Bigot, 1862 fait partie de la sous-famille des Apistomyinae.

Les larves et nymphes des Blépharocérides constituent, avec celles des Simuliides les éléments dominants des communautés torrenticoles car ce sont les formes les mieux adaptées à la vie dans les eaux rapides. On comprend que dans le cadre de recherches sur le peuplement et l'écologie des eaux courantes de l'île, j'aie été amené à établir la systématique préimaginale des sept espèces présentes en Corse.

Jusqu'à maintenant, les formes préimaginales de ces Diptères n'ont pas suscité d'études systématiques particulières; pourtant le présent travail montre que les états aquatiques des différentes espèces peuvent être distingués sans trop de difficultés en se référant à des caractères morphologiques simples. Une étude de ce type pourrait être étendue à l'ensemble de la faune européenne. 


\section{2. - ELEMENTS DE TAXONOMIE LARVAIRE ET NYMPHALE}

Avant 1963, date de ma première publication relative à ces Diptères, on ne connaissait pour la faune corse que la larve d'A. elegans, d'après la description originale d'EDwards, ainsi que la larve et la nymphe de $L$. cinerascens décrites depuis longtemps d'après du matériel d'Europe continentale.

J'expose ici les principaux caractères taxonomiques des larves et nymphes des sept espèces corses.

\subsection{Sous-famille des Apistomyinae. Apistomyia.}

L'espèce corse présente tous les principaux caractères du genre. Larve au stade IV (fig. 1) :

- antennes à 2 articles

- parapodes simples en massue, sans bâtonnet
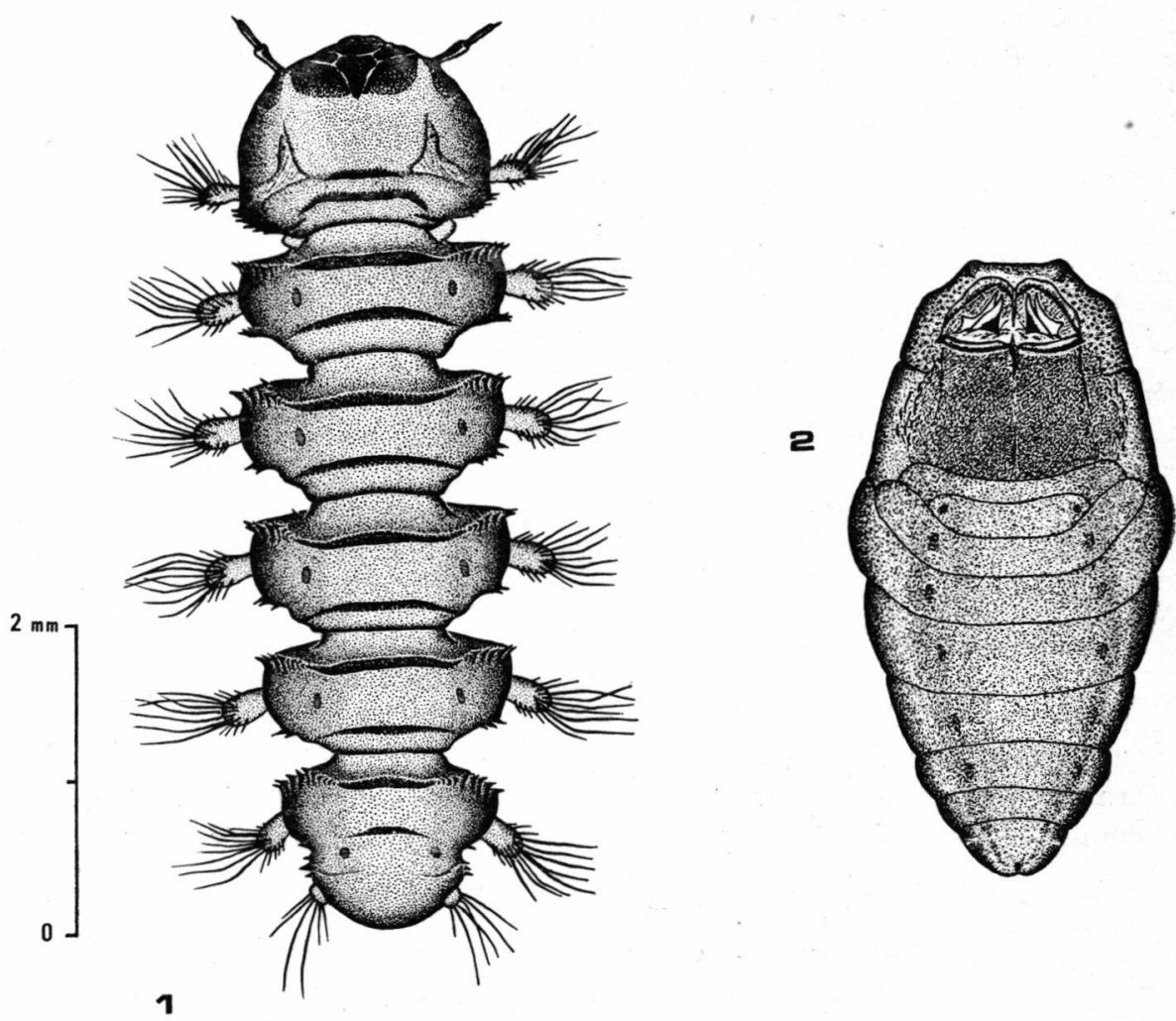

Fig. 1. - Larve d'Apistomyia elegans.

Fig. 2. - Nymphe d'Apistomyia elegans. 
- dernier segment larvaire pourvu de parapodes réduits (au total 7 paires de parapodes chez la larve)

- absence de segment intercalaire entre les 6 segments du corps

- tergites caractéristiques : les bords antérieur et postérieur sont marqués par un bourrelet sclérifié auquel fait suite latéralement une série d'épines

- branchies ventrales groupées en touffes de 5 filaments.

Nymphe (fig. 2).

Très remarquable par son appareil respiratoire formé de 2 lamelles internes, l'une triangulaire, l'autre à bords parallèles, et de 2 lamelles externes courtes et épaissies. Les lamelles externes du côté droit et celles du côté gauche se rejoignent sur la ligne médiodorsale du thorax et constituent ainsi une sorte de cadre chitineux délimitant une dépression au fond de laquelle sont insérés les 2 groupes de lamelles internes.

\subsection{Sous-famille des Blepharocerinae. Dioptopsis et Liponeura.}

\subsection{1. - Caractères génériques.}

Les larves des deux genres ont en commun les caractères suivants :

- parapodes larges à la base; présence d'un bâtonnet au dessus de chaque parapode

- présence d'un segment intercalaire entre les 6 segments du corps

- branchies ventrales groupées en touffes de 7 filaments.

Les larves se différencient par :

- des antennes courtes, à 2 articles chez Dioptopsis; longues, insegmentées et molles chez Liponeura

- des bâtonnets très allongés chez Dioptopsis; courts et peu visibles dorsalement chez Liponeura (fig. 3 et 5 à 9 ).

Chez les nymphes de Liponeura, l'appareil respiratoire est formé de 2 groupes de 4 lamelles dressées, de longueur à peu près égale. Les lamelles internes sont plus fines; les externes sont renforcées par un épaississement chitineux. A la base de chaque groupe se trouve une touffe serrée de filaments clairs, l' « organe basilaire», propre aux nymphes de ce genre. L'appareil respiratoire des nymphes de Dioptopsis (décrit pour la première fois à propos de $D$. vernus) est représenté de chaque côté du thorax par un groupe de 6 lamelles dressées. Les 2 lamelles externes sont courtes, larges et épaisses; à chacune d'elles est accolée vers l'intérieur une lamelle deux fois plus longue. Les deux lamelles les plus centrales sont très longues, étroites et de forme rubannée (fig. 4). 

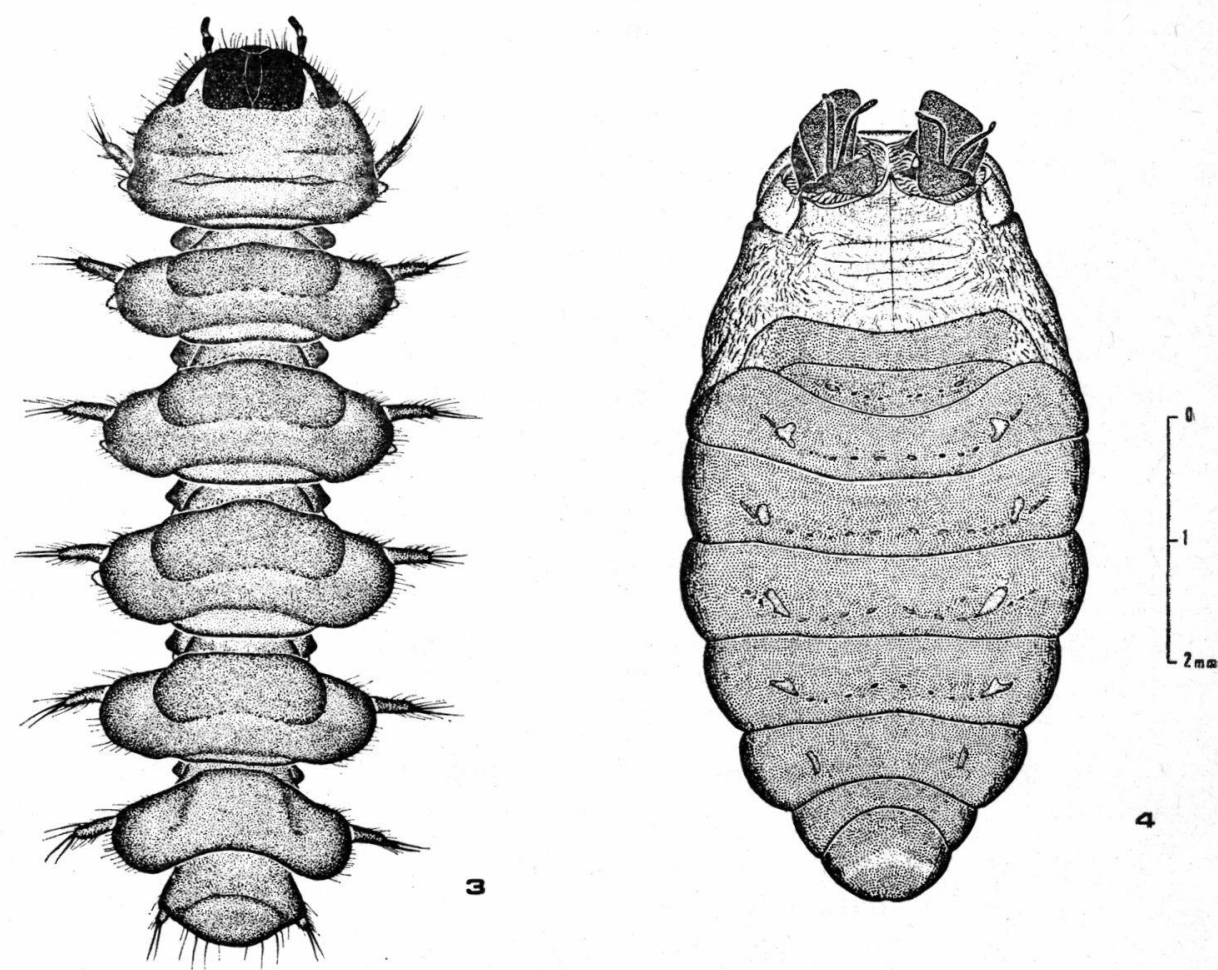

Fig. 3. - Larve de Dioptopsis vernus.

Fig. 4. - Nymphe de Dioptopsis vernus.

\subsection{2. - Caractères spécifiques.}

Parmi les cinq espèces de Liponeura de Corse, L. cinerascens, à tous les états de son développement, est morphologiquement isolée des autres espèces du genre. La larve et la nymphe ont été décrites dès 1912 par Hetschкo et, depuis, ces descriptions ont été plusieurs fois reprises et complétées [BISCHOFF 1928, KOMAREK et Vimmer 1934 Mannheims 1935]. Les quatre autres Liponeura constituent, compte tenu de leurs affinités morphologiques et phylogénétiques, deux groupes d'espèces affines : le groupe L. cortensisinsularis et le groupe L. edwardsiana-bischoffi.

- Les larves de $L$. cortensis et de $L$. insularis sont claires, sans pigmentation, si ce n'est une légère teinte grisâtre sur le dos de certains individus. 
Les larves de $L$. edwardsiana et de $L$. bischoffi présentent dorsalement, sur fond clair, des dessins gris-brun caractéristiques de ces deux espèces (fig. 5 et 6 ).

- Les larves de L. edwardsiana ont des antennes trois à quatre fois plus longues que le premier segment du corps; chez les larves de $L$. bischoffi le rapport entre ces deux longueurs est de 1,5, D'autre part, les dessins dorsaux sont caractéristiques pour chacune de ces deux espèces.

- Les larves de L. cortensis et de $L$. insularis se distinguent par la longueur relative des antennes : chez $L$. cortensis les antennes sont à peine plus longues que le premier segment du corps; chez L. insularis elles sont deux fois longues (fig. 7 et 8 ).

- Les nymphes de $L$. cortensis et de $L$. insularis ont leurs lamelles respiratoires dressées et recourbées vers le plan sagittal.

- Les nymphes de L. edwardsiana et de $L$. bischoffi ont leurs lamelles respiratoires dressées et verticales.

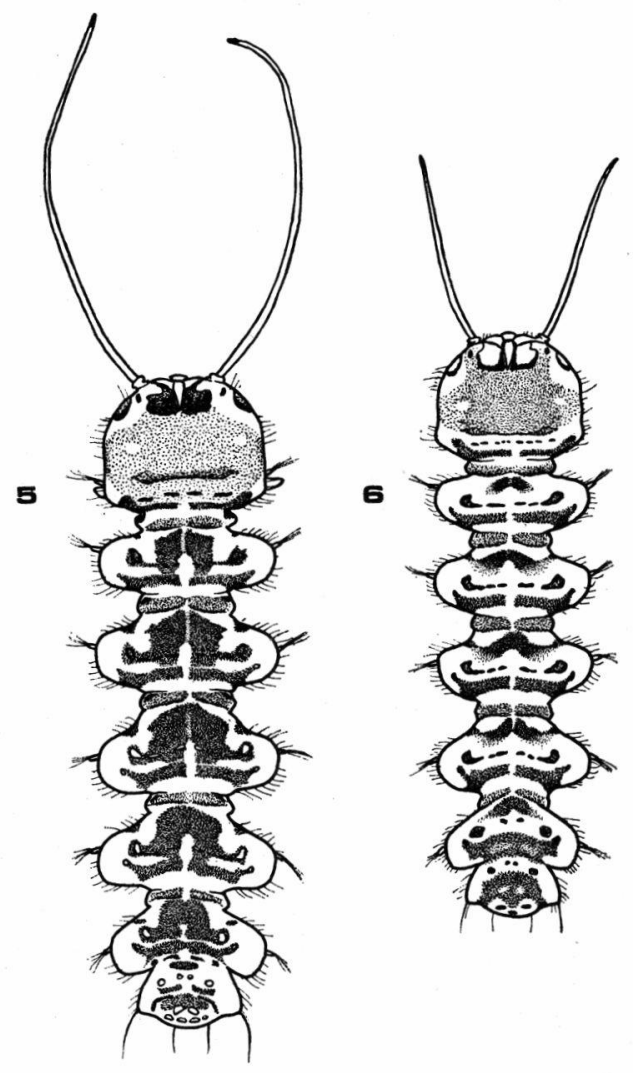

Fig. 5. - Larve de Liponeura edwardsiana.

Fig. 6. - Larve de Liponeura bischoffi. 
- Les nymphes de $L$. cortensis et de $L$. insulrais sont très semblables; le seul caractère qui permet de les différencier est représenté dans les figures 13 et 15 : en vue centrale, le tubercule antérieur apparaît plus saillant chez $L$. cortensis, plus effacé chez L. insularis.

- Chez les nymphes de $L$. edwardsiana, les lamelles internes de l'appareil respiratoire sont de moitié moins larges que les lamelles externes alors que chez $L$. bischoffi les lamelles internes sont plus larges (fig. 11, 12). De plus, le tubercule antérieur de la nymphè fournit un bon caractère de détermination : chez $L$. edwardsiana, il a la forme d'une cône surbaissé chez L. bischoffi il est bilobé (fig. 14 et 16 ).
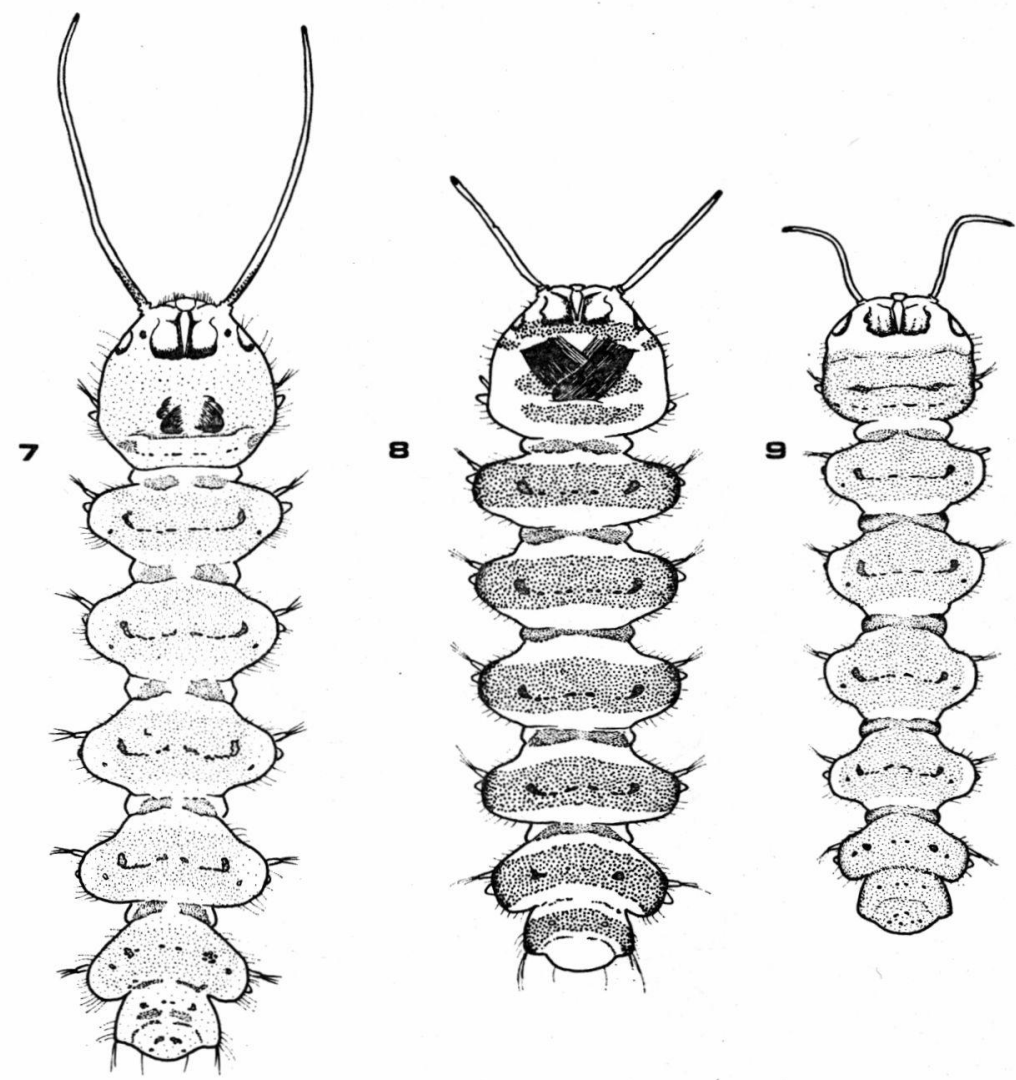

FIg. 7. - Larve de Liponeura insularis.

Fig. 8. - Prénymphe de Liponeura cortensis.

Fig. 9. - Larve de Liponeura cortensis. 

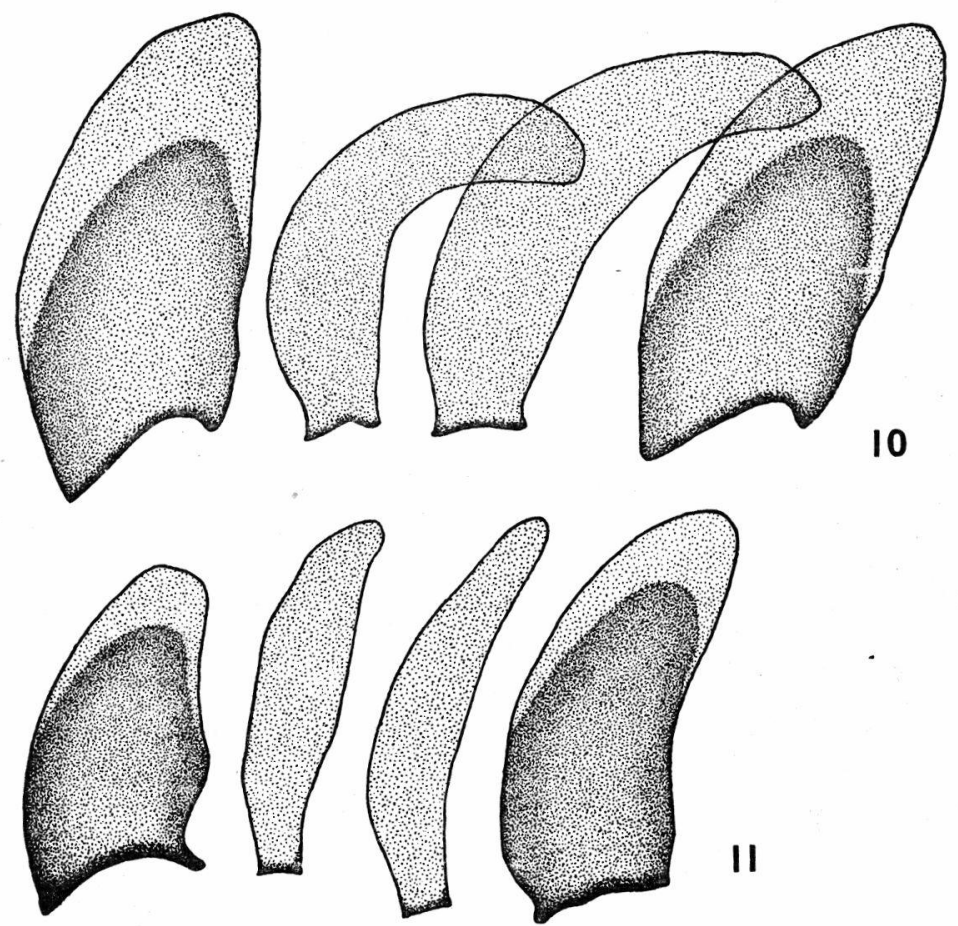

II
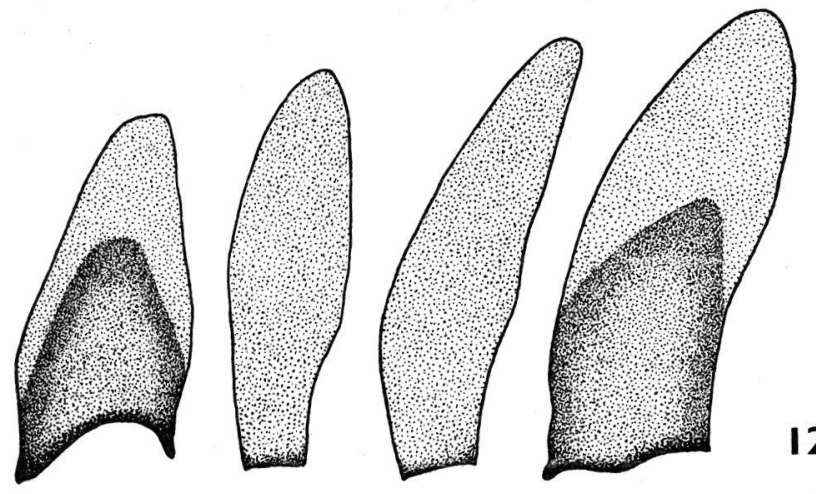

Fig. 10. - Lamelles respiratoires de la nymphe de Liponeura cortensis.

Fig. 11. - Lamelles respiratoires de la nymphe de Liponeura edwardsiana.

Fig. 12. - Lamelles respiratoires de la nymphe de Liponeura bischoffi. 


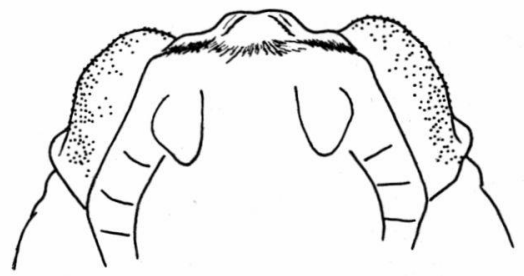

13

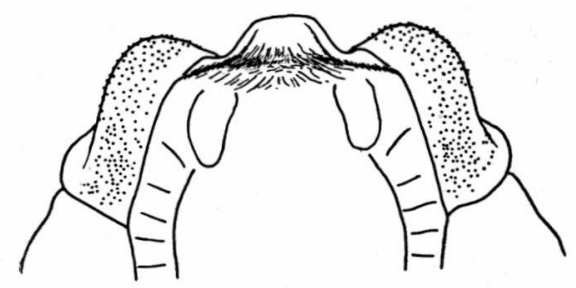

15

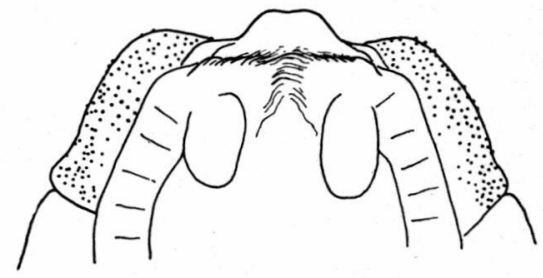

14

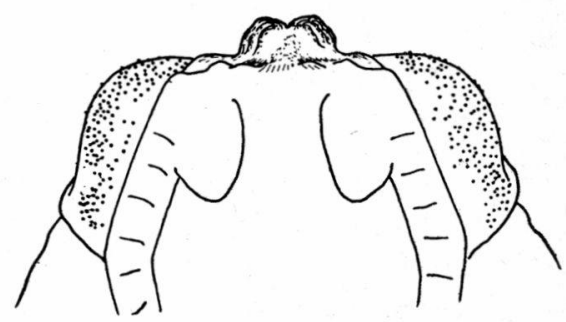

16

Fig. 13. - Région antérieure, en vue ventrale, de la nymphe de Liponeura insularis.

FIg. 14. - Région antérieure, en vue ventrale, de la nymphe de Liponeura edwardsiana.

Fig. 15. - Région antérieure, en vue ventrale, de la nymphe de Liponeura cortensis.

Fig. 16. - Région antérieure, en vue ventrale, de la nymphe de Liponeura bischoffi.

Fig. 17. - Euf de Liponeura insularis (vue dorsale).

Fig. 18. - Euf de Liponeura cortensis (vue dorsale).

FIg. 19. - Euf d'Apistomyia elegans (vue dorsale).

Fig. 20. - Euf de Dioptopsis vernus (vue dorsale). 


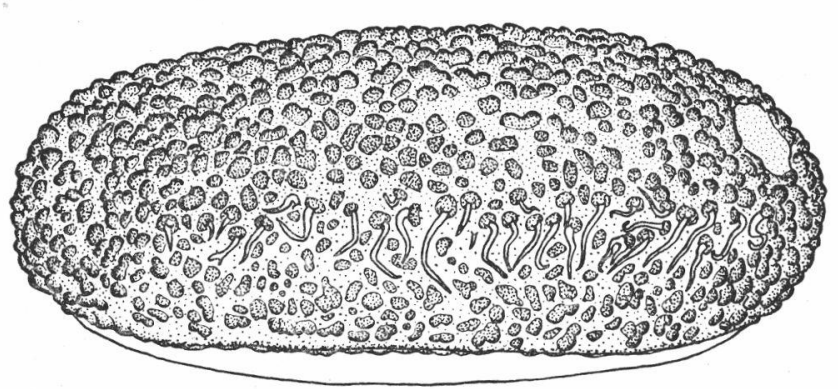

18

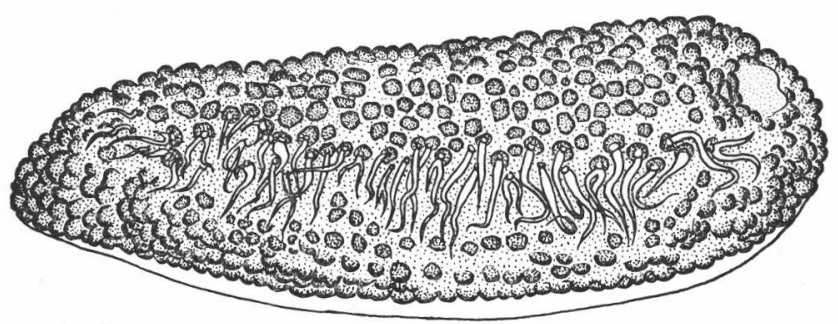

19

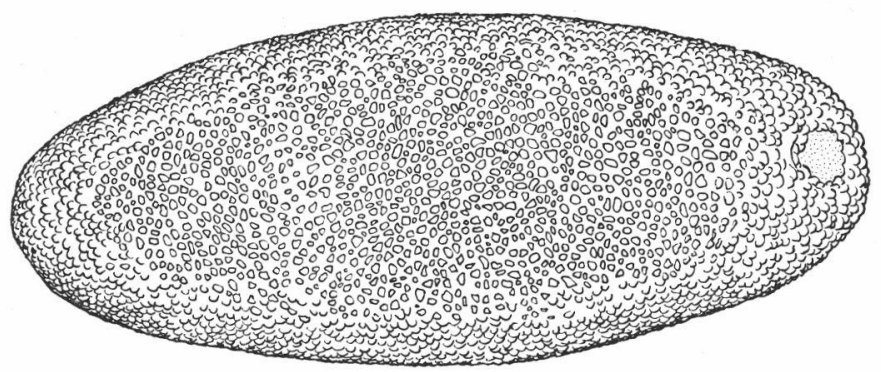

20

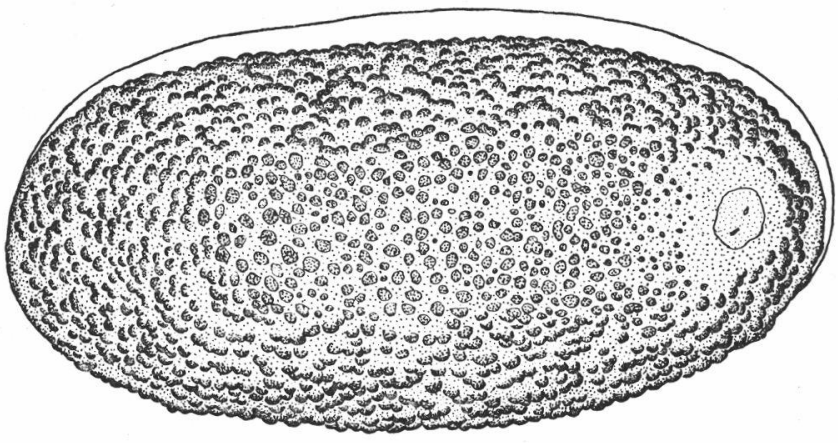



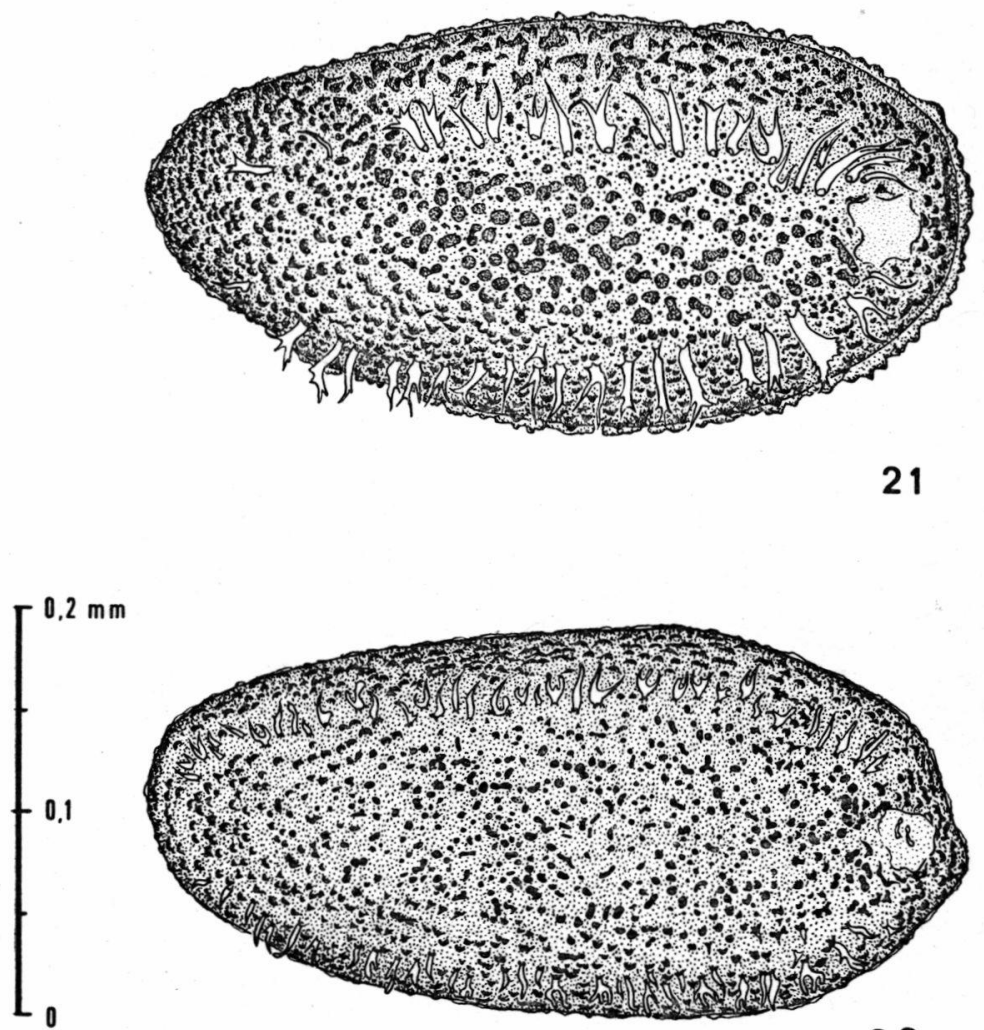

22

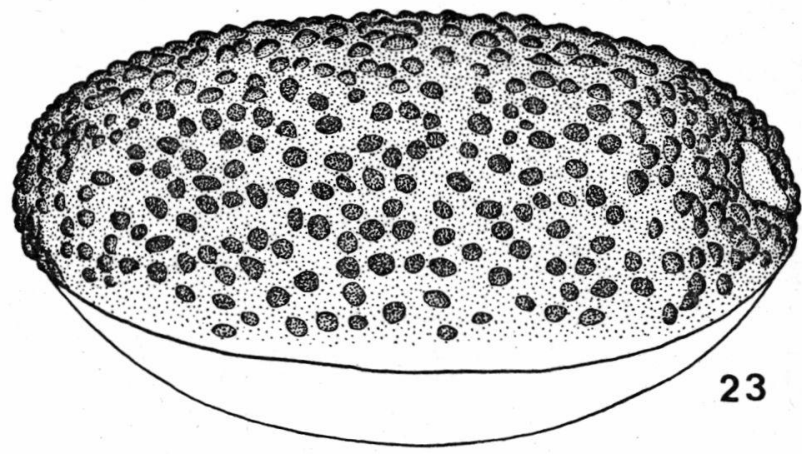

Fic. 21. - Euf de Liponeura edwardsiana (vue dorsale).

Fig. 22. - Euf de Liponeura bischoffi (vue dorsale).

FIg. 23. - Euf de Liponeura cinerascens (vue dorsale). 
Cette étude systématique, en dehors de son utilité pratique dans les recherches écologiques sur les eaux courantes, apporte des précisions complémentaires sur les relations entre certaines espèces d'un même genre. Ainsi, les affinités qui avaient été constatées précédemment [Giudicelli 1965], d'une part, entre les imagos de $L$. edwardsiana et $L$. bischoffi et, d'autre part, entre ceux de $L$. cortensis et $L$. insularis se trouvent confirmées au niveau des larves et des nymphes.

\section{3. - LES QEUFS}

Le même intérêt taxonomique se manifeste au stade de l'œuf. Pour les sept espèces de la faune corse les œufs montrent nettement, sur leur face dorsale épaisse et pigmentée, des caractères génériques et spécifiques, comme on peut le voir sur les figures 17 à 23 .

L'œuf de A. elegans est bien différent par sa forme et sa microsculpture de celui des autres espèces.

Les œufs de $L$. edwardsiana, $L$. bischoffi, L. insularis, L. cortensis sont remarquables par la présence de deux lignes de soies sur la face dorsale; celles-ci sont filiformes et pointues dans le groupe cortensis- insularis, courtes et laciniées dans le groupe edwardsianabischoffi.

Les cufs de $L$. cinerascens et de $D$. vernus ont plus de ressemblance entre eux qu'avec ceux des autres espèces. C'est peut-être là l'indice d'affinités entre les deux espèces; d'autant que $L$. cinerascens est isolée au sein du genre et pendant longtemps une appellation générique propre lui a été réservée.

Le genre Dioptopsis se situe juste en dessous du genre Liponeura dans l'arbre phylogénétique des Blépharocérides. Il est donc possible que $L$. cinerascens soit intermédiaire entre Dioptopsis et les autres espèces de Liponeura. L'étude des œufs d'un plus grand nombre d'espèces de Liponeura pourra apporter de nouveaux éléments d'appréciation.

\section{4. - NOTES ECOLOGIQUES}

La répartition des espèces a été étudiée en détail dans un réseau hydrographique de la Corse centrale situé entre 150 et $2000 \mathrm{~m}$ d'altitude. Ce réseau comprend la rivic̀re Restonica et ses branches collatérales ainsi que le fleuve Tavignano en aval de la petite ville de Corté (alt. $400 \mathrm{~m}$ ). 


\section{1. - Espèces expansives.}

L. cinerascens, L. edwardsiana, A. elegans ont une large distribution altitudinale et vivent dans des cours d'eau d'importance variable.

L. CINERASCEns (limite altitudinale : 400-1500 m).

C'est le seul Blépharocéridel qui a été trouvé dans les émissaires de sources à écoulement rapide. Ses formes aquatiques vivent dans l'hypocrenon et dans les trois zones du rhithron, ce qui correspond aux zones II, III, IV, V de la classification écologique des eaux courantes proposée par Illies et Botosaneanu [1963].

L. cinerascens est une forme psychrosténotherme. En hiver on la trouve aux altitudes inférieures et elle se nymphose au début du printemps dans des eaux dont la température varie de 5 à $7{ }^{\circ} \mathrm{C}$. En été, elle se cantonne dans les hautes vallées, au-dessus de $1200 \mathrm{~m}$ d'altitude, dans des eaux dont Ia température est inférieure à $15^{\circ} \mathrm{C}$.

L'espèce est univoltine; ses œufs passent par un état de diapause qui se produit à des époques différentes de l'année selon l'altitude.

L. EDWARDSIANA (limite altitudinale : 150-1 600 m).

Cette espèce présente la plus large répartition altitudinale. 'Très abondante au-dessous de $1000 \mathrm{~m}$, elle se raréfie à des altitudes supérieures. Son habitat comprend les trois zones du rhithron et l'épipotamon (zones III, IV, V et VI d'Illies et BotosaneaNu).

Elle présente dans l'année un nombre variable de générations selon l'altitude à laquelle évoluent ses population: : au-dessous de la cote 1000 l'espèce est polyvoltine, univoltine en amont. Dans l'épipotamon et l'hyporhithron (zones VI et V) entre 150 et $800 \mathrm{~m}$ d'altitude, $L$. edwardsiana a un développement continu comprenant trois générations par an. Entre 800 et $1000 \mathrm{~m}$ deux générations se succèdent en été; à la saison froide, pendant sept mois environ, on ne trouve plus ni larves, ni nymphes : le développement des œufs est alors arrêté. Au-dessus de la cote 1000 , la population alticole est univoltine et ne se manifeste que pendant trois mois d'été. Ainsi, cette espèce eurytherme s'adapte, par un phénomène. d'hibernation ou de diapause des œufs, aux conditions extrêmes de température qui règnent dans les hautes vallées pendant la saison froide. Les diverses modalités que réalise $L$. edwardsiana dans le développement de son cycle biologique doivent être interprétées comme des adaptations aux conditions d'environnement qui se modifient en altitude.

A. Elegans (limite altitudinale : 400-1 $500 \mathrm{~m}$ ).

Elle est caractéristique des trois zones du rhithron. Elle trouve son optimum écologique dans les torrents de l'épi- et du métarhi- 
thron (zones III et IV) où elle prolifère sur les rochers aspergés situés au pied des cascades. Son cycle évolutif est très semblable à celui de $L$. edwardsiana.

\section{2. - Espèces localisées.}

L. BISCHOFFI (répartition altitudinale : en dessous de $400 \mathrm{~m}$ ).

Espèce fluviatile, elle est limitée à l'épipotamon (zone VI). Elle se développe dans la partie des réseaux hydrographiques où les températures sont les plus élevées. Elle présente ses populations les plus abondantes en été, dans des eaux dont la température est égale ou supérieure à $25^{\circ} \mathrm{C}$.

On compte au moins trois générations par an. Les larves de la génération hivernale ont une évolution ralentie qui dure environ cinq mois; plusieurs générations se développent dans le courant de l'été et de l'automne.

L. INSULARIS (limite altitudinale : 400-1 $000 \mathrm{~m}$ ).

L. CORTENSIS (limite altitudinale $\$ 150-800 \mathrm{~m}$ ).

Ces deux espèces, appartenant au même groupe supra-spécifique, se remplacent d'amont en aval le long des cours d'eau. $L$. cortensis se cantonne dans le cours inférieur des rivières de montagne et dans les fleuves (hyporhithron et épipotamon $=$ zones $\mathrm{V}$ et VI); L. insularis se trouve dans le meta- et l'hyporhithron (zones IV et V). Dans la portion commune de leur aire de répartition, on trouve les deux espèces dans les mêmes stations, mais elles n'entrent pas en concurrence car leurs développements respectifs se font à des époques différentes. Les larves de $L$. insularis apparaissent au début de févrien celles de $L$. cortensis au début d'avril. Pour chaque espèce la nymphose a lieu trois à quatre mois plus tard; pendant le reste de l'année, les œufs subissent un arrêł de leur développement.

D. vernus (limite altitudinale : 400-1 $100 \mathrm{~m}$ ).

Les stades aquatiques habitent le méta- et l'hyporhithron. L'espèce est univoltine; les premières larves apparaissent en décembre et on observe les premières nymphes en avril dans des eaux dont la température est comprise entre 6 et $8^{\circ} \mathrm{C}$. D. vernus est une des espèces les plus sténothermes de la faune torrenticole. 


\section{RÉSUMÉ}

La faune corse comprend 7 espèces de Blépharocérides. Le présent travail porte sur les stades préimaginaux de ces espèces qui sont figurés et décrits. Les 7 espèces peuvent être identifiées, à l'aide de caractères morphologiques simples, à l'état de larves, de nymphes et d'œuts.

Pour chaque espèce l'auteur donne les principales caractéristiques autoécologiques : amplitude altitudinale, cycle vital, répartition en fonction du type de cours d'eau.

\section{PREIMAGINAL STAGES OF THE CORSICAN BLEPHAROCERIDAE (DIPTERA, NEMATOCERA). TAXONOMIC AND ECOLOGICAL STUDY.}

The corsican fauna includes 7 species of Blepharoceridae. The author presents a taxonomic study on larvae, pupae and eggs. The corsican species can be easily identified using external features.

Notes on ecology of individual species are given : altitudinal range, life cycle, distribution according to volume of flow.

\section{DIE PRÄ-IMAGO.STADIEN DER BLEPHAROCERIDEN (DIPTEREN, NEMATOCEREN) AUS KORSIKA. TAXONOMISCHE UND ÖKOLOGISCHE STUDIE}

Die Fauna Korsikas enthält 7 Blepharoceriden-Arten. Die vorliegende Arbeit beschäftigt sich mit den Prä-Imago-Stadien dieser Arten, welche abgebildet und beschrieben sind. An Hand von einfachen morphologischen Eigenschaften können die 7 Arten bereits im Larven- und Nymphenstadium sowie als Eier identifiziert werden.

Für jede Art gibt der Autor die wichtigsten auto-ökologischen Eigenschaften an : Höhenamplitude, Lebenszyklus, Verbreitung in Funktion der Strömung und in Abhängigkeit von der Art des Wasserlaufes.

\section{TRAVAUX CITÉS}

Bischoff (W.). 1928. - Die \&kologie der paläarktischen Blepharoceriden. Erg. Fortschr. d. Zool., 7 : 209-278.

Giudicelli (J.). 1965. - Nouvelle contribution à la connaissance des espèces récoltées en 1928 par F. W. Edwards. Ann. Soc. ent. Fr. (N. S.), 1 (2) : 363-371.

Illies (J.) et Botosaneanu (L.). 1963. - Problèmes et méthodes de la classiflcation et de la zonation écologique des eaux courantes considérées surtout du point de vue faunistique. Mitt. int. Verein. theor. angew. Limnol., 12 : 1-57.

Komarek (J.) et Vimmer (A.). 1934. - Blepharoceridae Balkanicae. Mitt. königl. Naturwiss. Inst. Sofia, 7 : 1-35.

Mannheims (B. J.). 1935. - Beitrage zur Biologie und Morphologie der Blepharoceriden. Zool. Forschr. Leipzig, $2: 1-115$.

(Laboratoire de Biologie Animale (Ecologie), Faculté des Sciences, Traverse de la Barasse, Saint-Jérôme, Marseille-13.) 